\title{
OUTCOMES AFTER SACRAL COLPOPEXY WITH NON-ABSORBABLE PERMANENT POLYPROPYLEN MESH - 5 YEARS EXPERIENCE
}

\author{
Morais Rodrigues, I.; Lermann, R.; Amaral, N.; Pereira, A.P.; Martins, A.; Veríssimo, C. \\ Obstetrics/Gynecology, Hospital Beatriz Ângelo, Loures, Lisbon, Portugal
}

$\checkmark$ Sacral colpopexy is a reconstructive surgical technique intended to repair pelvic organ prolapse (POP).

$\checkmark$ It is associated with lower risk of awareness of prolapse, repeat surgery for prolapse, postoperative urinary stress incontinence (USI) and dyspareunia than a variety of vaginal interventions.

$\checkmark$ Meshes currently available and used are lighter than those previously used but lack evidence of safety.

Aim: describe our experience and compare laparotomic and laparoscopic sacral colpopexy outcomes, including both naïve and post-hysterectomyzed women.

Retrospective, descriptive study - clinical records analysis

Comparision of complications between groups: Fisher exact Test; p-value $<0.05$

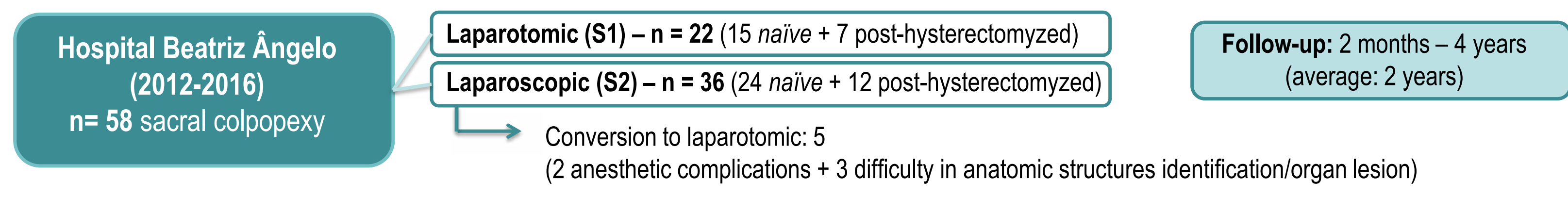

\begin{tabular}{|c|c|c|c|c|}
\hline \multicolumn{2}{|c|}{ GROUP CHARACTERISTICS } & S1 & $\$ 2$ & Total \\
\hline \multirow{2}{*}{ Ethnicity } & White & $17(85 \%)^{*}$ & $28(100 \%)^{*}$ & \\
\hline & Black & $3(15 \%)^{*}$ & 0 & \\
\hline \multirow{3}{*}{ Age } & Medium & 57 & 57 & 57 \\
\hline & Minimum & 43 & 34 & \\
\hline & Maximum & 67 & 72 & \\
\hline \multirow{3}{*}{$\begin{array}{l}\text { Weight (BMI, } \\
\left.\qquad \mathrm{kg} / \mathrm{m}^{2}\right)\end{array}$} & $<25$ & $5(23 \%)$ & $13(36 \%)$ & \\
\hline & $25-29.9$ & $12(55 \%)$ & $17(47 \%)$ & \\
\hline & $\geq 30$ & $5(23 \%)$ & $6(27 \%)$ & \\
\hline \multicolumn{2}{|c|}{ With active sexual life } & $15(68 \%)$ & $30(83 \%)$ & $45(78 \%)$ \\
\hline \multicolumn{2}{|c|}{ Multiparous } & $22(100 \%)$ & $36(100 \%)$ & \\
\hline \multicolumn{2}{|c|}{$\geq 3$ vaginal deliveries } & $4(18 \%)$ & $5(14 \%)$ & $9(15 \%)$ \\
\hline \multicolumn{2}{|c|}{ Operative vaginal deliveries } & $2(9 \%)$ & $8(22 \%)$ & $10(17 \%)$ \\
\hline \multicolumn{2}{|c|}{ Newborn $(s) \geq 4000 \mathrm{~g}$} & $4(18 \%)$ & $7(19 \%)$ & $11(19 \%)$ \\
\hline \multicolumn{2}{|c|}{ Chronic hypertension } & $4(18 \%)$ & $16(44 \%)$ & $20(34 \%)$ \\
\hline \multicolumn{2}{|c|}{ Diabetes Mellitus } & $2(9 \%)$ & $4(11 \%)$ & $6(10 \%)$ \\
\hline
\end{tabular}

\begin{tabular}{crr}
\hline $\begin{array}{c}\text { SIMULTANEOUS SURGICAL } \\
\text { PROCEDURES }\end{array}$ & \multicolumn{1}{c}{ S1 } & \multicolumn{1}{c}{ S2 } \\
\hline Hysterectomy & $15(68 \%)$ & $23(64 \%)$ \\
\hline USI surgery (TVT-0) & $2(9 \%)$ & $3(8 \%)$ \\
$\begin{array}{c}\text { Perineoplasty, anterior and/or } \\
\text { posterior colpoplasty }\end{array}$ & $11(50 \%)$ & $11(31 \%)$ \\
\hline
\end{tabular}

\begin{tabular}{|c|c|c|c|c|}
\hline \multicolumn{2}{|c|}{ TIMES } & \multicolumn{2}{|c|}{$\begin{array}{l}\text { Operative } \\
\text { (minutes) }\end{array}$} & \multirow{2}{*}{$\begin{array}{l}\text { Average hospitalization } \\
\text { (hours) }\end{array}$} \\
\hline \multirow{3}{*}{ s1 } & Total & $\Rightarrow$ & 165 & \\
\hline & naïve & & 175 & 80 \\
\hline & post hyst. & & 144 & 82 \\
\hline \multirow{3}{*}{ S2 } & Total & $\Rightarrow$ & 218 & 75 \\
\hline & naïve & & 232 & 71 \\
\hline & post hyst. & & 190 & 84 \\
\hline
\end{tabular}

$\longrightarrow$ The typical woman who underwent sacral colpopexy in our hospital was a caucasian, overweighted, 57-year-old women with active sexual life

\begin{tabular}{crrcc}
\hline $\begin{array}{c}\text { INTRAOPERATIVE } \\
\text { COMPLICATIONS }\end{array}$ & \multicolumn{1}{l}{ S1 } & \multicolumn{1}{l}{ S2 } & p-value & Total \\
\hline Anesthetic & 0 & $2(6 \%)$ & - & $2(3 \%)$ \\
\hline Bleeding & $2(9 \%)$ & $1(3 \%)$ & NS $(0.55)$ & $3(5 \%)$ \\
Organ lesion & $2(9 \%)$ & $2(6 \%)$ & NS $(0.63)$ & $4(7 \%)$
\end{tabular}

\begin{tabular}{|crrcr}
\hline $\begin{array}{c}\text { POSTOPERATIVE } \\
\text { COMPLICATIONS }\end{array}$ & S1 & S2 & p-value & Total \\
\hline $\begin{array}{c}\text { Relapse of symptomatic } \\
\text { POP }\end{array}$ & $6(27 \%)$ & $2(6 \%)$ & Sign $(0.04)$ & $8(14 \%)$ \\
\hline .. with need of surgery & $3(14 \%)$ & $1(3 \%)$ & NS $(0.14)$ & $4(7 \%)$ \\
\hline de novo USI & $2(9 \%)$ & $8(22 \%)$ & NS $(0.29)$ & $10(17 \%)$ \\
\hline Mesh extrusion & 0 & $1(3 \%)$ & - & $1(2 \%)$ \\
\hline $\begin{array}{c}\text { Wound infection/ } \\
\text { dehiscence }\end{array}$ & $1(5 \%)$ & $2(6 \%)$ & NS (1) & $3(5 \%)$ \\
\hline $\begin{array}{c}\text { Dyspareunia/ pelvic pain } \\
\text { Constipation }\end{array}$ & $1(5 \%)$ & $3(8 \%)$ & NS (1) & $4(7 \%)$ \\
\hline
\end{tabular}

$\checkmark$ Permanent mesh appears to be a safe and effective treatment of pelvic organ prolapse regardless of abdominal or laparoscopic approach.

$\checkmark$ Patient satisfaction is achieved with both approaches.

$\checkmark$ Relapse of symptomatic prolapse is higher with laparotomic; nevertheless, when considering need for surgical treatment there is no statistical difference.

$\checkmark$ Other main complications are similar between groups. 